\title{
Acceptability of Male Circumcision for Prevention of HIV/AIDS in Sub-Saharan Africa: A Review
}

\author{
N. Westercamp · R. C. Bailey
}

Published online: 20 October 2006

(C) Springer Science+Business Media, LLC 2006

\begin{abstract}
Based on epidemiological, clinical and experimental evidence, male circumcision (MC) could have a significant impact on the HIV epidemic in selected areas. We reviewed studies of the acceptability of MC in sub-Saharan Africa to assess factors that will influence uptake of circumcision in traditionally non-circumcising populations. Thirteen studies from nine countries were identified. Across studies, the median proportion of uncircumcised men willing to become circumcised was $65 \%$ (range $29-87 \%$ ). Sixty nine percent (47-79\%) of women favored circumcision for their partners, and $71 \%(50-90 \%)$ of men and $81 \%$ (70-90\%) of women were willing to circumcise their sons. Because the level of acceptability across the nine countries was quite consistent, additional acceptability studies that pose hypothetical questions to participants are unnecessary. We recommend pilot interventions making safe circumcision services available in conjunction with current HIV prevention strategies and evaluating the safety and acceptability of circumcision.
\end{abstract}

Keywords Male circumcision · Acceptability · HIV-1 · Africa

\section{Introduction}

Numerous observational studies have reported a significant protective effect of male circumcision (MC)

N. Westercamp · R. C. Bailey $(\bowtie)$

Division of Epidemiology and Biostatistics,

School of Public Health, University of Illinois at Chicago,

1603 W. Taylor Street, MC 923, Chicago, IL 60612, USA

e-mail: rcbailey@uic.edu against HIV and other sexually transmitted infections (STIs) in men (Bailey, Plummer, \& Moses, 2001; Cameron et al., 1989; Gray et al., 2000; Lavreys et al., 1999; Siegfried et al., 2003; Urassa, Todd, Boerma, Hayes, \& Isingo, 1997; Weiss, Quigley, \& Hayes, 2000). Recently, a randomized controlled trial (RCT) of MC to reduce HIV incidence in Orange Farm, South Africa was stopped prematurely due to an observed protective effect of MC of $60 \%$ in intention to treat analysis and $76 \%$ in a per protocol analysis. This effect was consistent with the protective effect found in cohort studies (Auvert et al., 2005).

Ecological studies have shown that the countries in sub-Saharan Africa with the highest HIV prevalence are those in which MC is little practiced (Halperin \& Bailey, 1999; Moses et al., 1990). Based on the epidemiological and experimental evidence to date, $\mathrm{MC}$ could have a significant impact on the HIV epidemic in these most highly affected countries. However, the effectiveness of the intervention will depend on many factors, not the least of which is the extent to which $\mathrm{MC}$ is accepted and taken up by males in these populations. If sufficient numbers of males are circumcised, there could be an effect similar to herd immunity since preventing men from becoming infected will also protect their sex partners. At more moderate levels of uptake, the effect is less clear.

In addition to the proportion of males who will become circumcised, the age at circumcision will also be a determinant of how rapidly the intervention results in reduction of HIV prevalence in the population. If infant circumcision is preferred over, say, pubertal circumcision, then the time lag from introduction of a large scale intervention until observable reductions in HIV prevalence could be decades. Because acceptance of MC 
by men and by parents of males in traditionally non-circumcising communities will be crucial to the success of a MC intervention for reducing HIV prevalence, we provide a review of the extant literature on acceptability of MC in sub-Saharan Africa.

\section{Study Collection and Search Strategies}

Criteria for inclusion in this review were established before the literature searches were carried out and included studies researching acceptability of MC as an HIV prevention method formally or as a part of a larger study, conducted in sub-Saharan Africa and published in a peer-reviewed journal or presented at an international conference. Electronic searches were conducted in MEDLINE using the following strategy: term "circumcision" in the title, abstract or keywords was combined with "acceptability", "attitudes" or "beliefs" in the title, abstract or keywords generating 920 articles, subset by "HIV" or "STIs/STD" in the title, abstract or keywords producing 244 articles, and finally limited to English language articles published from 1980 through 2006 resulting in 229 publications. Electronic search conducted in Google Scholar using phrase "acceptability of male circumcision in Africa as HIV prevention" resulted in 142 publications. Nine articles were directly related to the acceptability of circumcision in sub-Saharan Africa. Four additional studies were identified through personal communication with authors. A map of study sites in nine countries is presented in Fig. 1. Key characteristics of the 13 studies included in this review are shown in Table 1.

\section{Diversity of the Study Sample}

All studies employed some variation of a convenience sample. Out of 13 studies reviewed, eight were designed specifically to study acceptability of MC (Bailey, Muga, Poulussen, \& Abicht, 2002; Kebaabetswe et al., 2003; Lagarde, Dirk, Puren, Reathe, \& Bertran, 2003; Lukobo \& Bailey, Submitted; Mattson, Bailey, Muga, Poulussen, \& Onyango, 2005; Ngalande, Levy, Kapondo, \& Bailey, 2006; Scott, Weiss, \& Viljoen, 2005; Tsela \& Halperin, 2006), two included questions on MC acceptability in the context of a larger study (Bailey, Neema, \& Othieno, 1999; Halperin, Fritz, McFarland, \& Woelk, 2005), and three included formal MC acceptability data collection as well as previously collected data as part of a larger scope of research (Bailey, Unpublished report to AIDSMARK, 2002; Nnko, Washija, Urassa, \& Boerma, 2001; Rain-Taljaard et al., 2003). Seven of the studies were performed in largely ethnically homogenous populations (Bailey, Unpublished report to AIDSMARK, 2002; Bailey et al., 2002; Halperin et al., 2005; Mattson et al., 2005; Nnko et al., 2001; Scott et al., 2005; Tsela \& Halperin, 2006), while the remaining studies implemented specific strategies to ensure an ethnically mixed sample (Bailey et al., 1999; Kebaabetswe et al., 2003; Lagarde et al., 2003; Lukobo \& Bailey, Submitted; Ngalande et al., 2006; Rain-Taljaard et al., 2003). Ten of 13 studies included both male and female participants (Bailey, Unpublished report to AIDSMARK, 2002; Bailey et al., 2002; Kebaabetswe et al., 2003; Lagarde et al., 2003; Lukobo \& Bailey, Submitted; Mattson et al., 2005; Ngalande et al., 2006; Nnko et al., 2001; Rain-Taljaard et al., 2003; Scott et al., 2005). The remaining three studies were restricted to males (Bailey et al., 1999; Halperin et al., 2005; Tsela \& Halperin, 2006). Only two studies addressed acceptability in adolescent populations separately from adults (Nnko et al., 2001; Rain-Taljaard et al., 2003). Three studies purposely included the participation of female sex workers (Bailey et al., 2002; Lukobo \& Bailey, Submitted; Ngalande et al., 2006), and four studies included the opinions of MC providers in the assessment of circumcision acceptability/promotion (Bailey, Unpublished report to AIDSMARK, 2002; Bailey et al., 2002; Rain-Taljaard et al., 2003; Scott et al., 2005). Nine studies included both rural and urban populations (Bailey, Unpublished report to AIDSMARK, 2002; Bailey et al., 2002; Kebaabetswe et al., 2003; Lagarde et al., 2003; Lukobo \& Bailey, Submitted; Mattson et al., 2005; Ngalande et al., 2006; Nnko et al., 2001; Tsela \& Halperin, 2006); one study limited participation to rural groups only (Scott et al., 2005); and three studies were restricted to urban groups (Bailey et al., 1999; Halperin et al., 2005; Rain-Taljaard et al., 2003). All the studies were conducted in areas where circumcision is not traditionally practiced. Two purposely also included at least one area where most men are circumcised (Lukobo \& Bailey, Submitted; Ngalande et al., 2006). Ten studies assessed the circumcision status of male participants (Bailey, Unpublished report to AIDSMARK, 2002; Bailey et al., 1999; Halperin et al., 2005; Kebaabetswe et al., 2003; Lagarde et al., 2003; Mattson et al., 2005; Nnko et al., 2001; Rain-Taljaard et al., 2003; Scott et al., 2005; Tsela \& Halperin, 2006) and all studies allowed participation regardless of circumcision status.

\section{Summary of Quantitative Results of Acceptability}

Eight of the 13 studies reviewed included quantitative assessments of the acceptability of MC in six countries 


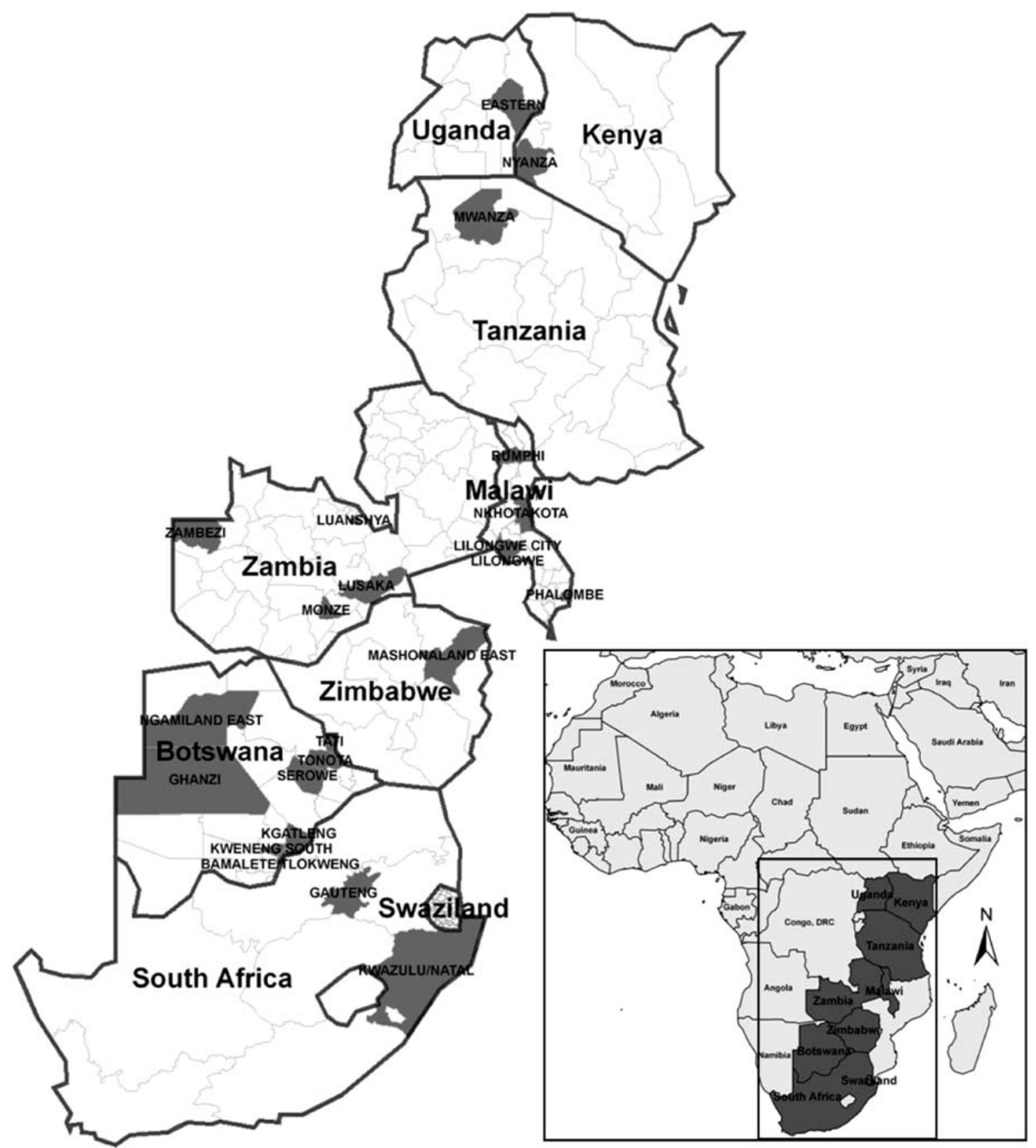

Fig. 1 Locations (by level 3 administrative unit) where male circumcision (MC) acceptability studies were conducted

using interview questionnaires. Results are summarized in Fig. 2 and Table 2. Four of the eight studies included women respondents. Willingness of uncircumcised men to become circumcised varied from $29 \%$ in Uganda to $87 \%$ in Swaziland. The variation depended in part on how the question was posed and the context of the study. For example, one of the highest acceptability levels (81\%) was recorded in Botswana after an informational session in which participants were told about the health benefits and risks associated with the procedure (Kebaabetswe et al., 2003). In some studies, adults were asked if they would be circumcised or prefer their partner to be circumcised "if MC were proven to be protective against HIV and STIs" (Halperin et al., 2005; Lagarde et al., 2003; Rain-Taljaard et al., 2003; Tsela \& Halperin, 2006). In others, participants were asked if they would accept MC "if it were safe and affordable" (Bailey et al., 1999; Kebaabetswe et al., 2003; Mattson et al., 2005; Scott et al., 2005).

In general, approximately the same proportion of women would prefer circumcision for their partners or their sons as men would prefer circumcision for 


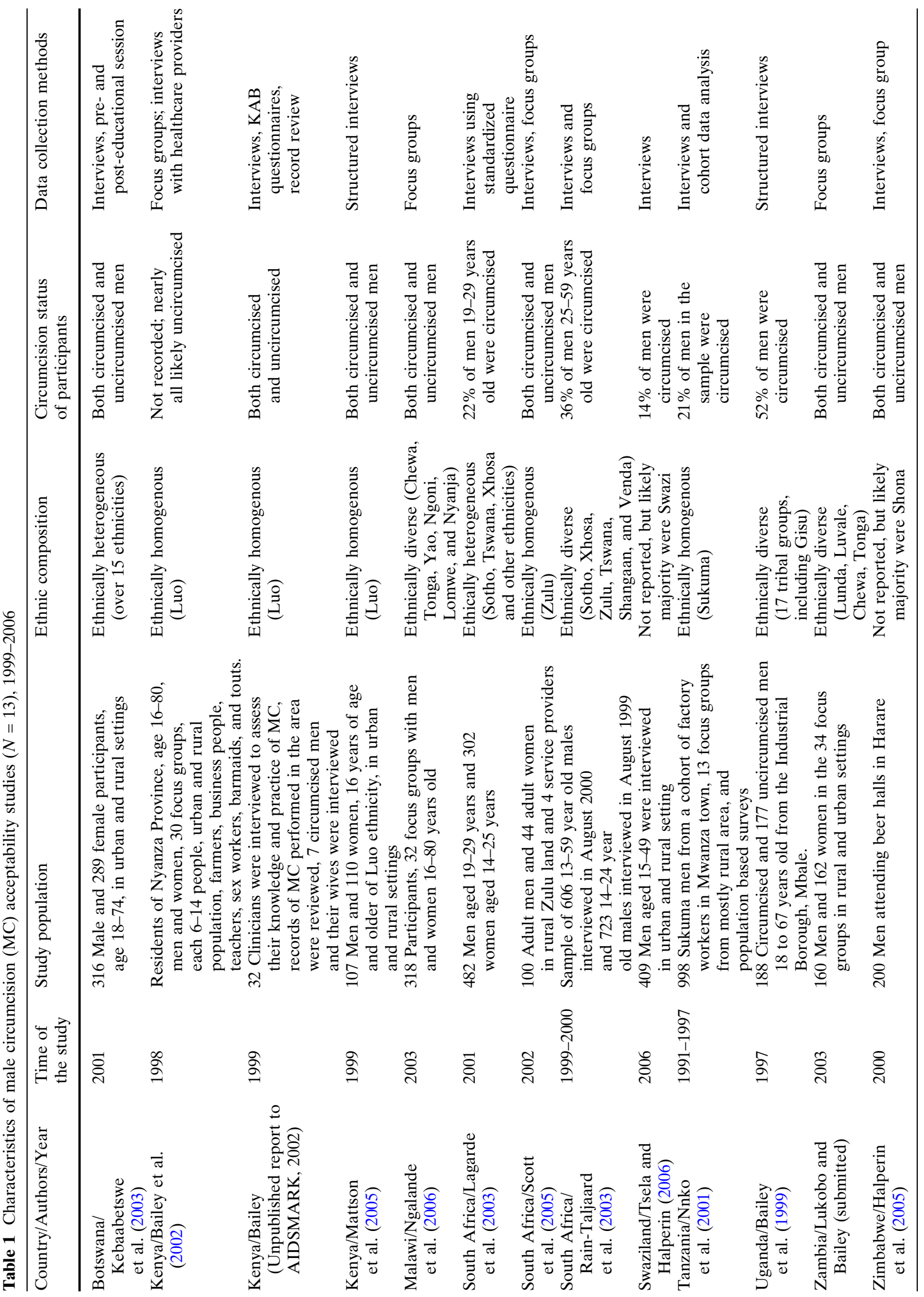




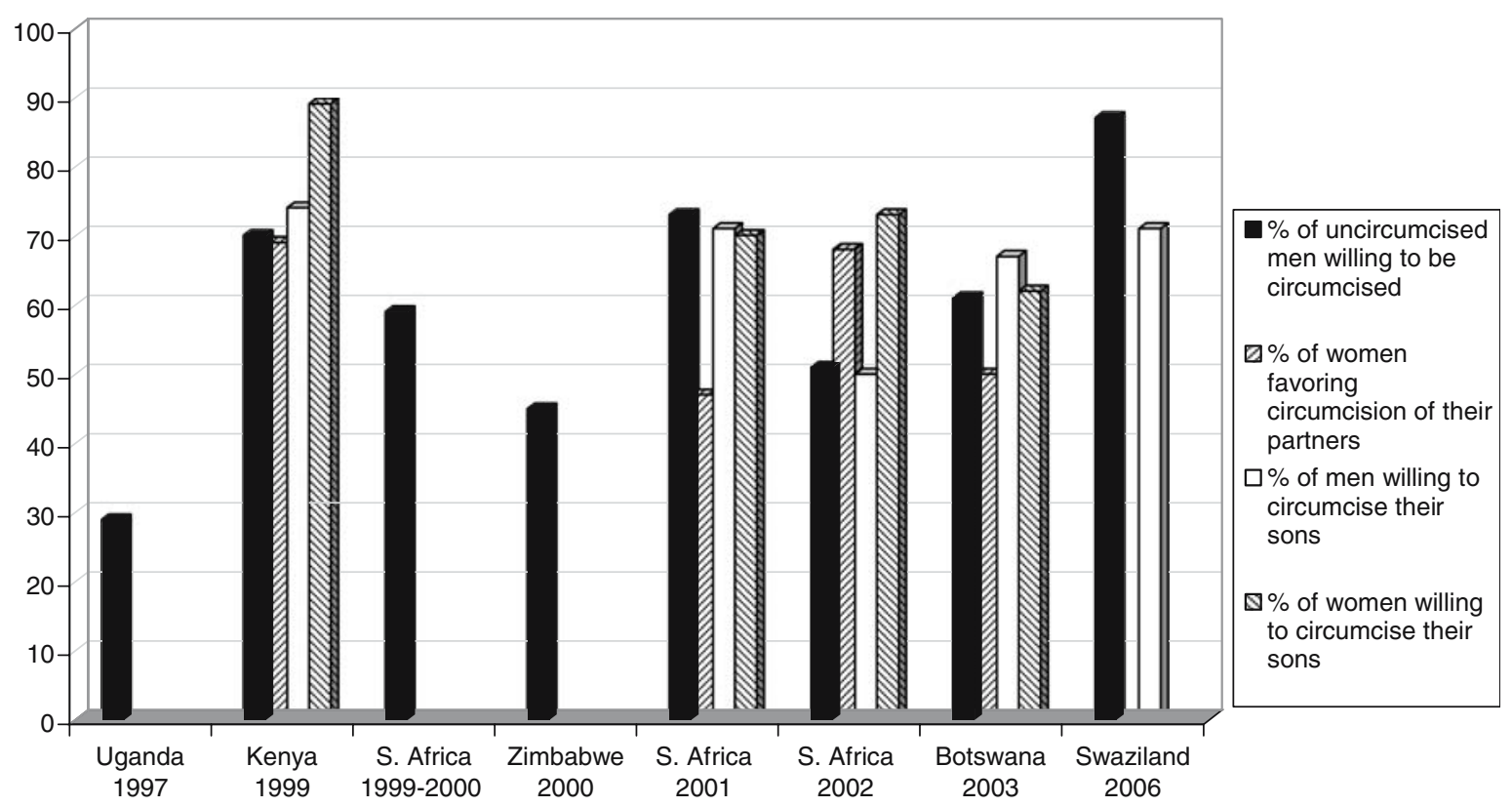

Fig. 2 Levels of male circumcision (MC) acceptability from eight quantitative studies in six sub-Saharan African countries

themselves or their sons. In Botswana, Kenya, South Africa and Swaziland, where men or women were asked about circumcision for their sons, more adults would agree to the procedure for their child than for their spouse or themselves. Approximately $75 \%$ of parents would seek circumcision for their son if it was safe, affordable and shown to be protective against HIV and STIs.

Across studies, the median proportion of uncircumcised men willing to become circumcised was $65 \%$ (range $29-87 \%$ ). Sixty nine percent (range $47-79 \%$ ) of women favored circumcision for their partners, and $71 \%(50-90 \%)$ of men and $81 \%(70-90 \%)$ of women were willing to circumcise their sons. The study restricted to rural population found that $51 \%$ of men were willing to become circumcised, while median proportion in the same category was $45 \%$ (range $29-59 \%)$ in three urban studies and $77 \%(70-87 \%)$ in studies that included both rural and urban population.

\section{Barriers to the Acceptability of MC}

\section{Pain}

Apprehension about pain during and after the procedure was reported to be the major barrier to $\mathrm{MC}$ acceptability in most studies (Bailey et al., 2002; Kebaabetswe et al., 2003; Lukobo \& Bailey, Submitted; Mattson et al., 2005; Ngalande et al., 2006; Scott et al., 2005). Participants belonging to non-circumcising ethnic groups were familiar with the circumcision practices in neighboring circumcising tribes where pain was a key characteristic of the procedure. As a rite of passage to becoming a man, the endurance of the pain from circumcision is often an integral aspect of the ceremony. For example, of 108 circumcised participants in South Africa, $42.6 \%$ described the traditional procedure as "very painful", $34.4 \%$ as "mildly painful", and $18.5 \%$ as "not painful" (Lagarde et al., 2003).

\section{Culture and Religion}

Lack of circumcision was mentioned as an element of the ethnic identity of those who do not circumcise traditionally. However, remaining with one's foreskin is not considered crucial to one's own ethnic identity. It serves as an ethnic marker primarily used by others. In both Botswana and Swaziland studies, only 2\% of participants, for example, felt that circumcision would lead to disapproval by their community (Kebaabetswe et al., 2003; Tsela \& Halperin, 2006), although in Botswana $22 \%$ cited "cultural reasons" as a factor in their decision not to circumcise their male child (Kebaabetswe et al., 2003). It is fundamentally different from belonging to an ethnic group that does practice traditional circumcision. For the Yao in Malawi, for example, or the Lunda and Luvale tribes in Zambia, or the Bagisu in Uganda (Bailey et al., 1999; Lukobo \& Bailey, Submitted; Nnko et al., 2001), it is unacceptable to remain uncircumcised, to the extent that forced circumcisions of older boys are not uncommon.

In some ethnic groups in which circumcision is not commonly practiced, disapproval of circumcision is 


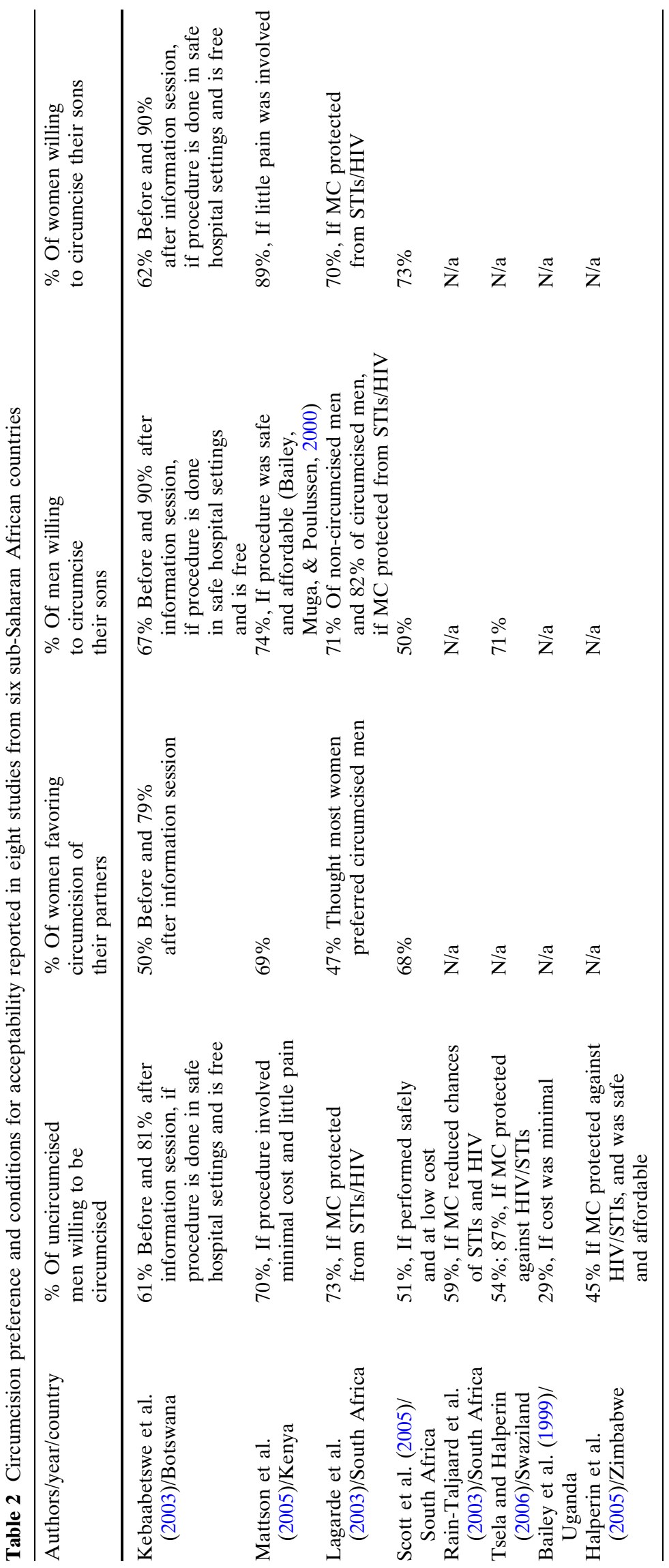


evident in the existence of a derogatory term for a circumcised man or a man with a congenitally shortened prepuce. These terms include "rayuom" in DhoLuo (Bailey et al., 2002) and "njilwa" in the Sukuma language (Nnko et al., 2001). In ethnically homogenous areas, circumcision could lead to rejection by local women and serve as a barrier to marriage (Bailey et al., 2002; Lukobo \& Bailey, Submitted). In more ethnically diverse areas, however, circumcision among traditionally non-circumcising peoples could be held as a positive, increasing a man's chances of being accepted by the women of the surrounding circumcising groups.

Religion is a major determinant of circumcision acceptability. MC is universally associated with Islam. It is also considered fundamental to some minority Christian and animist sects. There was no clear consensus on compatibility of MC with Christian beliefs (Bailey et al., 1999; Lukobo \& Bailey, Submitted; Ngalande et al., 2006; Nnko et al., 2001; Rain-Taljaard et al., 2003; Scott et al., 2005). Great variability in perceptions of Christian churches' positions on MC was described by different study populations, ranging from condemning $\mathrm{MC}$ as a pagan practice (RainTaljaard et al., 2003) to viewing MC as consistent with Christian tradition according to the Bible and Jesus' circumcision status (Lukobo \& Bailey, Submitted). In South Africa $38 \%$ of circumcised and $32 \%$ of uncircumcised study participants described circumcision as "forbidden" by their religion (Lagarde et al., 2003). Sukuma study participants in Tanzania felt that the Christian religion did not theologically promote MC, while circumcision services were known to be available in church-run hospitals (Nnko et al., 2001). Lukobo and Bailey describe the prevalent Zambian perception of circumcision being linked with Muslim or animist Chawa heritage, with several participants also reporting the belief that Christians should practice MC since Jesus was circumcised and the Bible teaches the practice (Lukobo \& Bailey, Submitted). Similar findings were reported by Ngalande et al. in Malawi (Ngalande et al., 2006). In Kenya the Nomiya Church and a few other small Christian sects require circumcision for church membership (Mattson et al., 2005).

Rain-Taljaard and colleagues report the South African belief that circumcision is fundamentally an African tradition and that Western ideas concerning the practice should not be taken seriously. Further, participants stated that many Christian churches opposed circumcision as a pagan tradition (Rain-Taljaard et al., 2003). However, it was unclear whether this opposition was directed at circumcision itself or at rites and ceremonies with which it was associated.
Before MC is promoted in a country, it would be prudent to consult and collaborate with religious leaders to learn the stance of the various churches regarding MC. In many cases, churches can act as helpful advocates or obstructive opponents and may have significant influence on acceptability of MC.

\section{Cost}

The cost of the procedure was a significant barrier to MC acceptability by participants in many studies (Bailey, Unpublished report to AIDSMARK, 2002; Bailey et al., 2002; Lagarde et al., 2003; Lukobo \& Bailey, Submitted; Mattson et al., 2005). Some participants expressed the opinion that if circumcision were promoted by the government, it should be provided at health clinics and hospitals for free or at reduced cost (Bailey et al., 2002; Lukobo \& Bailey, Submitted; Ngalande et al., 2006). Others recognized the need to pay for services because a free circumcision was viewed as being of potentially poor quality (Ngalande et al., 2006). Male and female participants in Zambia believed that, if the MC procedure were free or extremely inexpensive, more men would be willing to get circumcised (Lukobo \& Bailey, Submitted). In one study as many as $34 \%$ of participants who initially stated that their preference was to remain uncircumcised changed their minds when the proposed cost of the procedure was set at US\$3.00 (Mattson et al., 2005). Cost of traditional circumcision was considered to be high in many areas and there is a gradual shift from traditional to medical circumcision in part for this reason (Bailey \& Egesah, 2006; Lukobo \& Bailey, Submitted; Ngalande et al., 2006; Rain-Taljaard et al., 2003). Traditional circumcision is often expensive due to the costs of food, drink, special clothing and other items required during a sometimes prolonged celebration.

\section{Complications and Adverse Effects}

If men and parents believe that circumcision leads to high rates of complications, then uptake of MC is likely to be slow. Concerns for safety were universal in the studies examined. Mothers were vocal in their concerns, especially in cases of infant and early childhood circumcision. Excessive bleeding was a major concern and this fear was heightened if the procedure was to be performed by a traditional circumciser outside the hospital setting (Bailey et al., 2002; Lukobo \& Bailey, Submitted; Ngalande et al., 2006). Infection and difficulty in healing were expressed as concerns as well, but were generally believed to be minimized in clinical settings (Bailey et al., 2002; Lukobo \& Bailey, 
Submitted; Ngalande et al., 2006). Using the same knife for several boys was believed to be common in traditional settings and a source of infections, including HIV (Bailey et al., 2002; Halperin et al., 2005; Lagarde et al., 2003; Ngalande et al., 2006; Rain-Taljaard et al., 2003). Women were especially opposed to circumcision at the traditional initiation schools, as they feared that their children may be injured or die during the process (Rain-Taljaard et al., 2003).

Overall, there seemed to be a great deal of trust in medical practitioners and a strong preference for circumcision services to be made available in public health facilities by trained health professionals.

\section{Potential for Behavioral Disinhibition}

If men and their partners believe that circumcision offers protection from HIV infection, they may be less inhibited ("disinhibited") in their sexual activities and engage in higher HIV risk behaviors, thereby mitigating a partially protective effect of MC. Fortunately, the perception that $\mathrm{MC}$ provides full protection against HIV and STIs was found to be generally rare, but it was expressed by a few study participants in South Africa and in Nyanza Province, Kenya (Bailey et al., 2002; Rain-Taljaard et al., 2003). In focus groups in Kenya, Malawi and Zambia a concern about the possibility of behavioral disinhibition was inevitably expressed. Most participants did seem to appreciate the concept of risk reduction opposed to risk elimination (Bailey et al., 2002; Lukobo \& Bailey, Submitted; Ngalande et al., 2006). Similarly in Swaziland $87 \%$ of study participants advocated having only one partner and $94 \%$ promoted condom use for circumcised men (Tsela \& Halperin, 2006).

There is some evidence of behavioral disinhibition among circumcised men. A study in South Africa found a significant association between circumcision status and the higher reported number of non-spousal lifetime partners (Lagarde et al., 2003). Circumcised men in Uganda were found to engage in more HIV risk behaviors than uncircumcised men (Bailey et al., 1999). In addition to reporting more extramarital partners in the previous year $(1.13$ vs. $0.62, P<0.01)$, circumcised men had an overall higher "risk profile". A few respondents in another South African study expressed the belief that MC potentially encouraged adultery as newly circumcised men were curious to test the new shape of the penis (Rain-Taljaard et al., 2003).

One study in Botswana found that participants felt that circumcision before the age of six years may help to avoid a change in sexual behavior associated with sense of increased protection due to circumcision
(Kebaabetswe et al., 2003). Men attending beer halls in Harare, Zimbabwe were aware of the partial protection against HIV provided by MC, and had a good understanding of the limitations and the concept of risk reduction (Halperin et al., 2005).

\section{Other Reasons Not to Circumcise}

Other barriers to circumcision, mentioned by participants, were lack of access to health care, required time away from work, the loss of penile sensitivity, reduction in penis size, decreased ability to satisfy women, excessive sexual desire, increased promiscuity (Bailey et al., 2002; Rain-Taljaard et al., 2003), and the perception of circumcision as old-fashioned (Lagarde et al., 2003; Rain-Taljaard et al., 2003).

\section{Facilitators of MC Acceptability}

\section{Hygiene}

Penile hygiene was universally recognized as being extremely important and was viewed as a major benefit of circumcision (Bailey et al., 2002; Halperin et al., 2005; Kebaabetswe et al., 2003; Lukobo \& Bailey, Submitted; Mattson et al., 2005; Ngalande et al., 2006; Nnko et al., 2001). A great majority of participants, both male and female from multiple studies, agreed that it was much easier for a circumcised man to maintain cleanliness (Bailey et al., 2002; Lukobo \& Bailey, Submitted; Mattson et al., 2005; Ngalande et al., 2006; Nnko et al., 2001; Rain-Taljaard et al., 2003).

The majority of participants, including women, believed that it was worrisome that men do not maintain proper hygiene. Because women were the primary providers of water, poor penile hygiene was often seen as a woman's failing (Bailey et al., 2002; Lukobo \& Bailey, Submitted). In both Zambia and Malawi women were considered responsible for cleaning their partners' penises after sexual intercourse. Additionally, women in these populations linked their own risk of STIs to their partners' genital hygiene (Lukobo \& Bailey, Submitted; Ngalande et al., 2006). Ease of maintaining proper penile hygiene proved a major factor in women's acceptability of circumcision (Bailey et al., 2002; Lukobo \& Bailey, Submitted; Mattson et al., 2005; Ngalande et al., 2006).

\section{Protection from STIs and HIV}

Hygiene as a mechanism of protection from STIs was mentioned by a great number of participants (Bailey 
et al., 2002; Lukobo \& Bailey, Submitted; Ngalande et al., 2006). It was held that germs, dirt, bacteria, and viruses had a greater opportunity to proliferate in the warm moist environment beneath the foreskin (Bailey et al., 2002; Lukobo \& Bailey, Submitted; Ngalande et al., 2006; Nnko et al., 2001; Rain-Taljaard et al., 2003). Participants also expressed a belief that it would be easier to detect rashes and/or ulcerations with the foreskin removed allowing for earlier treatment (Bailey et al., 2002; Ngalande et al., 2006). The foreskin was also perceived as a portal of entry for sexually transmitted infection as the tissue is considered prone to traumatic injury during sexual intercourse (Bailey et al., 2002; Lukobo \& Bailey, Submitted; Ngalande et al., 2006; Rain-Taljaard et al., 2003). MC was recognized as a medical procedure to reduce or eliminate penile ulcerations and diseases of the penis (Bailey et al., 2002; Lukobo \& Bailey, Submitted; Ngalande et al., 2006; Nnko et al., 2001). Conversely, a minority of respondents in Zambia reported that the circumcised penis was "always dry", "susceptible to cracking", and that this state provided a portal of entry for bacteria and viruses (Lukobo \& Bailey, Submitted).

Seventy percent of Botswana study participants willing to circumcise their male child listed protection from STIs or HIV among their reasons for doing so (Kebaabetswe et al., 2003). In Nyanza Province, Kenya, $79 \%$ of uncircumcised men and $81 \%$ of women believed that it was easier for uncircumcised men to acquire STIs compared to circumcised men. This belief dropped to $43 \%$ and $60 \%$, respectively, concerning the acquisition of AIDS (Mattson et al., 2005). In Swaziland, $81 \%$ of participants stated that MC reduced risk of STIs and $18 \%$ believed that MC reduced risk of HIV (Tsela \& Halperin, 2006). In Tanzania STIs were considered more severe and more infective in uncircumcised men, with ulcers healing faster in those who are circumcised (Nnko et al., 2001). Nearly all commercial sex workers believed that there exists a strong association between lack of circumcision and STIs, including HIV (Ngalande et al., 2006). In South Africa (Scott et al., 2005), no association was found between willingness to be circumcised and perceived health benefits. It was belief about sexual pleasure that was the strongest predictor of being willing to undergo circumcision.

\section{Acceptability by Other Ethnic Groups}

Common reasons given for favoring $\mathrm{MC}$ were the social, political, and sexual benefits that could accrue when interacting with those in predominantly circumcising groups (Bailey et al., 2002; Lukobo \& Bailey, Submitted; Ngalande et al., 2006). The Luo believed that they were often discriminated against by other Kenyans due to their circumcision status which led to political exclusion and even security concerns in times of social upheaval (Bailey et al., 2002). Many younger men from traditionally non-circumcising groups cited being accepted as a sexual or marriage partner by women from other ethnic groups as an important reason to be circumcised (Bailey et al., 2002; Lukobo \& Bailey, Submitted; Ngalande et al., 2006; Nnko et al., 2001).

\section{Sexual Pleasure Among Circumcised versus Uncircumcised}

How circumcision is perceived to influence sexual drive, sexual performance, and sexual pleasure for the man himself or for his partner is likely to influence decision making around MC. Participants in many studies believed that circumcision enhances sexual pleasure (Bailey, Unpublished; Bailey et al., 2002; Lagarde et al., 2003; Lukobo \& Bailey, Submitted; Mattson et al., 2005; Ngalande et al., 2006; Nnko et al., 2001; Rain-Taljaard et al., 2003).

Most studies assessed three factors associated with sexual activity based on circumcision status: sexual performance, sexual pleasure for men, and sexual pleasure for women. Fifty percent of circumcised and $30 \%$ of uncircumcised participants in South Africa believed that MC increased sexual performance, while only $21 \%$ and $14 \%$, respectively, believed that MC decreased sexual pleasure (Lagarde et al., 2003). Other studies found that a high proportion of men and a majority of women believed that circumcised men enjoyed sex more than uncircumcised men (Mattson et al., 2005; Rain-Taljaard et al., 2003). About half of female participants reported preference for circumcised men (Lagarde et al., 2003; Mattson et al., 2005). Many had no preference. A study in South Africa found that men were 8 times more likely to prefer circumcision if they believed that circumcised men enjoyed sex more, and 6 times more likely to prefer circumcision if they believed that women enjoy sex more with circumcised men (Scott et al., 2005). Other studies did not find a consensus about circumcision status and sexual pleasure on the part of the man or the woman (Bailey et al., 2002; Lukobo \& Bailey, Submitted; Ngalande et al., 2006). For some, circumcision was irrelevant to pleasure, as pleasure was more related to emotional attachment and past sexual experience (Lukobo \& Bailey, Submitted; Ngalande et al., 2006). Attitudes about circumcision and pleasure may 
be different in areas where dry sex is practiced (Lukobo \& Bailey, Submitted).

\section{Other Reasons to Circumcise}

Other reasons to be circumcised reported by participants included the belief that it was easier for circumcised men to use condoms (Bailey, Unpublished report to AIDSMARK, 2002; Bailey et al., 2002; Kebaabetswe et al., 2003), that MC proved manhood, that aim during urination was improved, and that not being circumcised brought bad luck (Rain-Taljaard et al., 2003).

Time and Setting of Circumcision Procedure

\section{Preferred Age at Circumcision}

The ages at which males become circumcised will have an effect on how rapidly MC interventions may impact the HIV epidemic in any given area. Preferred age at circumcision varied both between and within studies. There appeared to be two leading directions exhibited by many studies: either circumcise males as babies due to a simpler procedure, less fear, easier care, and faster healing, or circumcise males around puberty and adolescence when boys can decide and take care of the wound for themselves (Bailey et al., 2002; Lukobo \& Bailey, Submitted; Ngalande et al., 2006; Rain-Taljaard et al., 2003; Scott et al., 2005).

Among the nine countries where acceptability studies have been undertaken, only in Botswana were most participants in favor of circumcision in infancy and early childhood. Fifty-five percent of respondents were in favor of circumcising children under 6 years old with half of those preferring neonatal circumcision (Kebaabetswe et al., 2003). In all other areas a significant minority were in favor of infant or early childhood MC, but most favored circumcision between ages $8-16$ years with very few saying that over 18 years was best. Those who advocated for infant circumcision did so for reasons relating to decreased pain during the procedure and faster healing times (Bailey et al., 2002; Lukobo \& Bailey, Submitted), although babies under 1 year of age were thought to experience excessive pain, leading to crying and fevers (Lukobo \& Bailey, Submitted). Participants from Malawi viewed infants especially vulnerable to potential complications of MC due to "lack of maturity" and difficulty of timely detection of bleeding due to babies being carried on the mothers' backs (Ngalande et al., 2006).
Many studies reported strong beliefs among participants that circumcision should take place before the onset of sexual activity (Lukobo \& Bailey, Submitted; Ngalande et al., 2006; Rain-Taljaard et al., 2003). Ages 7-13 years were thought to be best since the boy could make the decision for himself, understand the significance of the event, take care of the wound himself, heal faster than if done post-pubertally, and has likely not begun sexual activity (Bailey et al., 2002; Lukobo \& Bailey, Submitted; Ngalande et al., 2006; Rain-Taljaard et al., 2003).

Circumcision as an adult or post-pubertally was reported by many to be undesirable due to higher risk of complications, pain during the procedure (Ngalande et al., 2006; Rain-Taljaard et al., 2003), and painful erections after MC, leading to complications and delays in healing (Bailey et al., 2002; Lukobo \& Bailey, Submitted; Ngalande et al., 2006).

Many people from traditionally non-circumcising communities felt that they had insufficient knowledge to make a decision about when best to circumcise. They preferred to consult clinical professionals to get their advice (Bailey et al., 2002; Ngalande et al., 2006). Practitioners interviewed in Kenya and Malawi preferred not to perform neonatal circumcision due to the small size of the penis and foreskin, potentially leading to higher rates of errors and complications. These providers preferred to perform the operation at ages 8-12 years (Bailey et al., 2002).

\section{Preferred Circumcisers}

In areas where traditional circumcision is uncommon, the preference is overwhelmingly for a medical practitioner to be the provider. All studies reported fear of infection, bleeding, excessive pain, and possible mutilation at the hands of traditional circumcisers (Kebaabetswe et al., 2003; Lagarde et al., 2003; Lukobo \& Bailey, Submitted). In Zambia (Lukobo \& Bailey, Submitted), even in the traditionally circumcising area of Zambezi District, the majority believed medical doctors to be experienced, more apt to use sterile equipment, able to minimize pain through anesthesia, and capable of dealing with complications. The few participants who preferred traditional surgeons viewed these practitioners as more experienced and more willing to maintain confidentiality (Lukobo \& Bailey, Submitted).

Scott et al. found that $77 \%$ of male Zulu preferred MC by a doctor or medical surgeon, $8 \%$ by a nurse, $11 \%$ by traditional circumciser, and $3 \%$ by other providers (Scott et al., 2005). Another study based in South Africa observed that MC was commonly 
performed in both "initiation schools" and by clinical providers. The more common circumcision was in an ethnic group, the less likely it was done in medical settings (Rain-Taljaard et al., 2003).

Acceptability in Certain Populations

\section{Women's Beliefs and their Influence}

The influence of women on the decision to circumcise is likely to be highly variable across cultures and across families within communities. However, in many settings, women, as mothers and as partners, are likely to have considerable influence, even if it is not overt. Any effort to promote MC will be more successful if it appeals to women as well as men.

Bailey et al. (2002) found that women's beliefs may have a strong influence on male acceptability of circumcision in western Kenya. This influence may stem from women's strong emphasis on penile hygiene for their partners, and the wish to protect their young sons from acquisition of infections as they become sexually active. Scott et al. (2005), on the other hand, suggested that in South Africa women are likely to have only an indirect influence through the male perception that women enjoy sex more with circumcised men. A different study from South Africa found that women had a strong influence on men's decision to circumcise, often scheduling the appointment for their boyfriends or husbands. Single mothers, however, were believed to have no influence over their teenage sons' decisions to circumcise (Rain-Taljaard et al., 2003). Thirteen percent of circumcised participants in yet another South African study (Lagarde et al., 2003) reported undergoing circumcision because their partner expressly requested it.

\section{Acceptability in Youth}

Two out of thirteen studies assessed acceptability of MC among adolescents. In Tanzania, school aged boys and girls believed that it was easier for an uncircumcised man to acquire STIs, that it was easier for a circumcised man to maintain proper genital hygiene, and that circumcision enhanced sexual pleasure for both partners (Nnko et al., 2001). As in most areas, adolescent boys linked circumcision with modernity and good hygiene. Overall, adolescent males and females proved to be knowledgeable about potential benefits of MC (Nnko et al., 2001; Rain-Taljaard et al., 2003). Nnko et al. (2001) observed that knowledge and a positive attitude about MC became most obvious in secondary schools due to the effects of increased ethnic mixing.
Many studies found that younger participants were more likely to view circumcision favorably than their elders (Lukobo \& Bailey, Submitted; Mattson et al., 2005; Ngalande et al., 2006; Nnko et al., 2001). In Botswana, only $43 \%$ of men ages $45-59$ years were willing to be circumcised, compared to $65 \%$ of 25-34 year-olds (Kebaabetswe et al., 2003). In Kenya, younger men were more likely to accept circumcision. Among those $16-21$ years old, $71 \%$ said that they would prefer to be circumcised; whereas only $56 \%$ of those over 21 years preferred to be circumcised (Bailey, 2001). Results from more qualitative studies entailing focus group discussions were consistent with these quantitative results. Younger men in Zambia, Malawi and Tanzania were more likely to express a desire to be circumcised (Lukobo \& Bailey, Submitted; Ngalande et al., 2006; Nnko et al., 2001).

\section{Hypothetical versus Actual Acceptability}

Asking people whether they might prefer to be circumcised under various hypothetical scenarios (e.g., if it is found to reduce risk of HIV acquisition; or if it is at minimal cost and safe) is one means of assessing acceptability. A more realistic means is to discover where MC services are available and see who takes advantage of the services. Alternatively, one can offer the services in non-circumcising communities and see the response. This approach permits assessment of not just numbers seeking the services, but also the ages and population segments that respond as well as factors that inhibit or facilitate uptake of the services.

A trial intervention in Siaya District, Kenya-an area where circumcision is not traditionally practiced-was introduced in 1999 (Bailey, Unpublished report to AIDSMARK, 2002). During a 25 month period, 433 circumcisions were performed in health facilities where only 6 procedures had been done in the previous year. In a comparison district, where no intervention was available, just 24 circumcisions were preformed over the same period. Demand for MC services was judged to be high but was highly dependent on cost. When the price charged for a circumcision was reduced from $\$ 3.62 \mathrm{US}$ to $\$ 1.45$ US, demand surged, and $50 \%$ of all circumcisions occurred during the 2 months when the price was reduced. The median age of those circumcised was 18 years; $25 \%$ were below age 12 years, and an estimated $35 \%$ were circumcised before their sexual debut. The researchers felt that a greater number of younger males would have been circumcised had parental permission not been required for those under age 18 years and if the cost were reduced permanently, since older 
males tended to have more financial support (Bailey, Unpublished report to AIDSMARK, 2002). The results from this trial intervention are consistent with results from studies of hypothetical acceptability indicating that cost is consistently found to be a major barrier to uptake of circumcision in traditionally non-circumcising communities.

Further evidence of acceptability comes from the one RCT completed in Orange Farm, South Africa and the two ongoing trials in Kisumu, Kenya and Rakai, Uganda, both of which have completed enrollment. Because every participant in these three trials stands a $50 \%$ chance of being circumcised immediately upon randomization, all of them must prefer to be circumcised in order to enroll in the study. The Orange Farm trial screened 3,483 young men, ages $18-24$ years. We do not know what proportion of the total population of 18-24 year olds these men represent. However, that all but $203(5.8 \%)$ of the men consented to enroll indicates that acceptability was high (Auvert et al., 2005). In Kisumu, Kenya, a community in which $90 \%$ of adult men are uncircumcised, 6,686 of the 34,200 (19.5\%) uncircumcised men in the population ages 18-24 years came to the study clinic seeking to enroll in the study. Of these, 4,489 (67.1\%) were eligible to enroll, and of those eligible, $68.5 \%$ accepted to be randomized (Bailey, 2006). This acceptability rate agrees very closely with the $70 \%$ figure found in the sample by Mattson et al. (2005) from the same area. In Rakai, Uganda, a rural community in which $83 \%$ of adult men are uncircumcised, approximately $45 \%$ of all eligible HIV uninfected men in the community enrolled in the trial before enrollment was closed (R. Gray, personal communication). That such large numbers of men are willing to join these trials suggests that circumcision acceptability is high and that uptake of MC in these communities could be rapid, if sufficient resources are available to accommodate large numbers of procedures.

\section{Discussion}

Through searching electronic databases and contacting authors, we identified 13 studies from nine countries that include investigation of the acceptability of MC in traditionally non-circumcising regions in sub-Saharan Africa. We found one additional report of a pilot intervention introducing $\mathrm{MC}$ services into health facilities where circumcision was little practiced. The level of acceptability across the nine countries appears greater than might be expected, considering that all thirteen communities where the studies were per- formed were all traditionally non-circumcising. The lowest level of acceptability by uncircumcised men (29\%) was reported from eastern Uganda in a study conducted in 1997, before MC became well recognized as possibly being associated with STIs and HIV (Bailey et al., 1999). More than half of men in the regions studied appear to be receptive, if not eager, to become circumcised.

Cost, fear of pain, and concern for safety were the three most consistent barriers to acceptability of MC. In communities where circumcision is the norm families expect to incur the obligatory circumcision expenses negating the importance of cost. In noncircumcising communities circumcision is regarded as a voluntary procedure that may be unlikely to take precedence over competing needs. Cost is viewed as including not only the payment for the procedure, but also the opportunity costs of time away from work and other income generating activities. Cost as a primary consideration was shown dramatically by the pilot intervention in Siaya, Kenya, where men came in large numbers when the charges were lowered to $\$ 1.45 \mathrm{US}$ (Bailey, Unpublished report to AIDSMARK, 2002). These results indicate that the true cost of the procedure will have to be supplemented to achieve significant uptake of MC.

The concerns for safety and pain are based partially on the perception of circumcision as a surgical procedure with inherent risks and partially on the occasional press releases publicizing mutilations and deaths. Personal knowledge of neighboring communities where traditional initiates withstand excruciating pain also likely plays a role. Sustained uptake of MC will require performance of the procedure with minimal adverse events. This can be achieved through proper training and supervision of practitioners, proper instrumentation and sterilization, complete instructions to patients, follow-up with patients, and over all attention to quality control (Krieger et al., 2005).

The studies we reviewed revealed that it is virtually universal that Africans equate circumcision with improved hygiene. Also widespread is the belief that circumcision leads to reduced incidence of STIs achieved through improved hygiene, reduction in the number and severity of scratches, tears and abrasions to which the foreskin is susceptible and through earlier detection of ulcers, leading to earlier treatment. Although not as frequent, a significant proportion of participants in the studies also saw circumcision leading to reduced risk of HIV acquisition through the same route. If $\mathrm{MC}$ is proven in the remaining two clinical trials to reduce incidence of HIV and some STIs (e.g., HPV, HSV-2, chancroid and gonorrhea), 
this information will be consistent with the already existing beliefs of most sub-Saharan Africans.

Cultural norms, ethnic identity, and religious affiliation were viewed as central factors in acceptability of circumcision. Circumcision was associated with specific traditionally circumcising communities and with Muslims and members of a few minority Christian and animist sects. It will likely be important that confidentiality is maintained by circumcision practitioners, since stigmatization for being circumcised is a possibility in non-circumcising communities. An important conclusion reached by several studies was that circumcision was increasingly an issue of personal choice rather than ethnic identity (Rain-Taljaard et al., 2003; Scott et al., 2005). Urbanization, ethnic mixing, and exposure to other cultures and religions are conducive to higher acceptability of circumcision in traditionally non-circumcising ethnic groups.

In East and Southern Africa most MCs are done between ages 8 and 21 and the preferences for age at circumcision found in studies are consistent with these practices. However, a large enough proportion of people, especially mothers, preferred infant circumcision to consider making infant circumcision an available option. This should be an important consideration in designing $\mathrm{MC}$ interventions.

Information campaigns may be effective in increasing acceptability of MC. This was found to be true in Botswana and South Africa (Kebaabetswe et al., 2003; Scott et al., 2005). However, many studies demonstrated that both knowledge and acceptability of MC varies considerably by region within the same country. Therefore, informational campaigns may be more effective if targeted to particular communities.

Just as the international health community is concerned about the possibility that promotion of circumcision could lead to increases in risky sexual behavior (World Health Organization, 2005), participants in many of the studies reviewed were similarly concerned. Higher risk behaviors have been found to be associated with circumcision status previously in Uganda, Rwanda and Kenya (Bailey et al., 1999; Seed et al., 1995; Tyndall et al., 1996), as well as in the Orange Farm RCT (Auvert et al., 2005). This underlines the importance of the counseling and education that must be provided to men who undergo circumcision, reinforcing the idea of MC reducing, not eliminating, the risk of HIV and other STIs.

There are several limitations to the studies that we reviewed. All used convenience sampling to recruit participants. The results could be biased if recruits were more likely to participate if they had a favorable view of MC. This may not be a concern, since most studies had nearly $100 \%$ participation by those who were asked to participate. Only two studies verified the circumcision status of the participants (Lagarde et al., 2003; Nnko et al., 2001) and none of the studies verified MC status of partners of interviewed women. The direction in which this may have biased results is not clear. There were differences across studies in design: some were more qualitative with open ended questions asked in a group discussion setting, others were more quantitative using closed-ended questions during a one-on-one interview. There was variation in the wording of questions to participants about the conditions under which they would accept circumcision. Some studies were geographically restricted and, as a result, may have limited generalizability and lack of representiveness of populations. Geographical coverage was spotty within study countries, and some high HIV prevalence countries where MC is little practiced (e.g., Mozambique, Lesotho, Namibia) were not included. Lastly, there was variation in the time when the studies were conducted (range 1991-2006). Attitudes toward circumcision assessed by early studies (Bailey et al., 1999; Nnko et al., 2001) may have changed since the time of the study.

All studies attempted to assess peoples' beliefs and attitudes toward circumcision and their willingness to be circumcised under some hypothetical conditions sometime in the future. We cannot know from these studies what the actual uptake of circumcision would be if it were found to be protective in three clinical trials and was actively promoted. We have only one example of an introduction of MC services in a traditionally non-circumcising community (Bailey, Unpublished report to AIDSMARK, 2002), and this was at a time when circumcision could not be actively promoted, but could only be made available. Results from that intervention were instructive in that demand for safe circumcision was robust, but depended very much upon price.

The results from the thirteen available studies of acceptability of MC in nine countries in sub-Saharan Africa where circumcision is little practiced are very consistent. Acceptability of MC is likely to be high enough to have a significant impact on HIV prevalence in these communities, if $\mathrm{MC}$ is proven to have a protective effect similar to that found in observational studies and in the Orange Farm RCT. It is doubtful, given the consistency of results to date, that we will learn a great deal more by additional acceptability studies that pose hypothetical questions to participants. Instead, we recommend pilot interventions making circumcision services available in health facilities after training of clinicians and provision of proper 
instruments and supplies. There are reports that demand for MC services is already high in many traditionally non-circumcising communities in East and southern Africa (Bangre, 2006; Nnko et al., 2001; PlusNews, 2006; Timberg, 2005). There is a danger that this increasing demand will be filled by unqualified practitioners causing unnecessary adverse events. Pilot interventions will serve simultaneously to test whether there truly is a growing niche and, if so, to gain experience in filling the niche with safe, affordable services. At the same time, much will be learned about the operational requirements for training, instrumentation, safety, counseling and follow-up of patients, supervision of staff, monitoring of behavioral disinhibition, and about how MC services can be integrated with HIV/STIs prevention services, including VCT, STIs diagnosis and treatment, behavioral counseling, condom promotion and anti-retroviral therapies.

Acknowledgments We are grateful to Christine Mattson for her assistance with bibliography and to Daniel Halperin and George Schmid for their helpful comments.

\section{References}

Auvert, B., Taljaard, D., Lagarde, E., Sobngwi-Tambekou, J., Sitta, R., \& Puren, A. (2005). Randomized, controlled intervention trial of male circumcision for reduction of HIV infection risk: The ANRS 1265 trial. PLoS Medicine, 2(11), $1-11$.

Bailey, R. C. (2001). Male circumcision as an effective HIV prevention strategy: Current evidence. Paper presented at the 8th conference on Retroviruses and opportunistic infections. Chicago, IL, USA.

Bailey, R. C. (2006). Presentation to NIAID Data Safety Monitoring Board (DSMB), June 27, 2006.

Bailey, R. C., \& Egesah, O. (2006). Assessment of clinical and traditional male circumcision services in Bungoma District, Kenya: Complications rates and operational needs.

Bailey, R. C., Muga, R., \& Poulussen, R. (2000). Trial intervention introducing male circumcision to reduce HIV/STD infections in Nyanza province, Kenya: Baseline results. XIII International AIDS Conference, Durban, SA.

Bailey, R. C., Muga, R., Poulussen, R., \& Abicht, H. (2002). The acceptability of male circumcision to reduce HIV infections in Nyanza Province, Kenya. AIDS Care, 14(1), 27-40.

Bailey, R. C., Neema, S., \& Othieno, R. (1999). Sexual behaviors and other HIV risk factors in circumcised and uncircumcised men in Uganda. Journal of Acquired Immune Deficiency Syndrome, 22(3), 294-301.

Bailey, R. C., Plummer, F. A., \& Moses, S. (2001). Male circumcision and HIV prevention: Current knowledge and future research directions. The Lancet Infectious Diseases, $1(4), 223-231$.

Bangre, H. (2006). Swaziland: recrudescence de circoncision pour contrecarrer le sida? Afrik.com, March 15, 2006. http://www.afrik.com/article9595.html last opened March 22, 2006.
Cameron, D. W., Simonsen, J. N., D'Costa, L. J., Ronald, A. R., Maitha, G. M., Gakinya, M. N., Cheang, M., Ndinya-Achola, J. O., Piot, P., Brunham, R. C., et al. (1989). Female to male transmission of human immunodeficiency virus type 1: Risk factors for seroconversion in men. Lancet, 2(8660), 403-407.

Gray, R. H., Kiwanuka, N., Quinn, T. C., Sweankambo, N. K., Serwadda, D., Wabwirem-Mangen, F., Lutalo, T., Nalugoda, F., Kelly, R., Meehan, M., Chen, M. Z., Li, C., \& Wawer, M. J. (2000). Male circumcision and HIV acquisition and transmission: Cohort studies in Rakai, Uganda. AIDS, 14(15), 2371-2381.

Halperin, D. T., \& Bailey, R. C. (1999). Male circumcision and HIV infection: Ten years and counting. Lancet, 354, 1813-1815.

Halperin, D. T., Fritz, K., McFarland, W., \& Woelk, G. (2005). Acceptability of adult male circumcision for sexually transmitted disease and HIV prevention in Zimbabwe. Sexually Transmitted Diseases, 32(4), 238-239.

Kebaabetswe, P., Lockman, S., Mogwe, S., Mandevu, R., Thior, I., Essex, M., \& Shapiro, R. L. (2003). Male circumcision: An acceptable strategy for HIV prevention in Botswana. Sexually Transmitted Infections, 79(3), 214-219.

Krieger, J. N., Bailey, R. C., Opeya, J., Ayieko, B., Opiyo, F., Agot, K., Parker, C., Ndinya-Achola, J. O., Magoha, G. A. O., \& Moses, S. (2005). Adult male circumcision: Results of a standardized procedure in Kisumu District, Kenya. BJU International, 96(7), 1109-1113.

Lagarde, E., Dirk, T., Puren, A., Reathe, R. T., \& Bertran, A. (2003). Acceptability of male circumcision as a tool for preventing HIV infection in a highly infected community in South Africa. AIDS, 17(1), 89-95.

Lavreys, L., Rakwar, J. P., Thompson, M. L., Jackson, D. J., Mandaliya, K., Chohan, B. H., Bwayo, J. J., Ndinya-Achola, J. O., \& Kreiss, J. K. (1999). Effect of circumcision on incidence of human immunodeficiency virus type 1 and other sexually transmitted diseases: A prospective cohort study of trucking company employees in Kenya.[see comment]. Journal of Infectious Diseases, 180(2), 330-336.

Mattson, C. L., Bailey, R. C., Muga, R., Poulussen, R., \& Onyango, T. (2005). Acceptability of male circumcision and predictors of circumcision preference among men and women in Nyanza Province, Kenya. AIDS Care, 17(2), 182-194.

Moses, S., Bradley, J. E., Nagelkerke, N. J. D., Ronald, A. R., Ndinya-Achola, J. O., \& Plummer, F. A. (1990). Geographical patterns of male circumcision practices in Africa: Association with HIV seroprevalence. International Journal of Epidemiology, 19(3), 693-697.

Ngalande, R., Levy, J., Kapondo, C., \& Bailey, R. C. (2006). Acceptability of male circumcision for prevention of HIV infection in Malawi. AIDS and Behavior, 10(4), 377-385.

Nnko, S., Washija, R., Urassa, M., \& Boerma, J. T. (2001). Dynamics of male circumcision practices in northwest Tanzania. Sexually Transmitted Diseases, 28(4), 214-218.

PlusNews. (2006). Zambia: A snip in time can save lives. PLUSNEWS, March 21, 2006. www.plusnews.org/AIDSreport.asp?ReportID=5780 last opened March 21, 2006

Rain-Taljaard, R. C., Lagarde, E., Taljaard, D. J., Campbell, C., MacPhail, C., Williams, B., \& Auvert, B. (2003). Potential for an intervention based on male circumcision in a South African town with high levels of HIV infection. AIDS Care, 15(3), 315-327.

Scott, B. E., Weiss, H. A., \& Viljoen, J. I. (2005). The acceptability of male circumcision as an HIV intervention among a rural Zulu population, Kwazulu-Natal, South Africa. AIDS Care, 17(3), 304-313. 
Seed, J., Allen, S., Mertens, T., Hudes, E., Serufilira, A., Carael, M., Karita, E., Van de Perre, P., \& Nsengumuremyi, F. (1995). Male circumcision, sexually transmitted disease, and risk of HIV. Journal of Acquired Immune Deficiency Syndromes \& Human Retrovirology, 8(1), 83-90.

Siegfried, N., Muller, M., Volmink, J., Deeks, J., Egger, M., Low, N., Weiss, H., Walker, S., \& Williamson, P. (2003). Male circumcision for prevention of heterosexual acquisition of HIV in men. Cochrane Database of Systematic Reviews, 3, CD003362.

Timberg, C. (2005). In Swaziland, science revives an old rite. Washington Post December 26, 2005. www.washiungtonpost.com/wp-dyn/content/article/2005/12/25/

AR2005122500749.html last opened March 21, 2006.

Tsela, S., \& Halperin, D. T. (2006). Knowledge, attitudes and practices regarding male circumcision in the Manzini (central) region of Swaziland. In The XVI international AIDS conference. Toronto, Canada.

Tyndall, M. W., Ronald, A. R., Agoki, E., Malisa, W., Bwayo, J. J., Ndinya-Achola, J. O., Moses, S., \& Plummer, F. A. (1996).
Increased risk of infection with human immunodeficiency virus type 1 among uncircumcised men presenting with genital ulcer disease in Kenya. Clinical Infectious Diseases, 23(3), 449-453.

Urassa, M., Todd, J., Boerma, T., Hayes, R., \& Isingo, R. (1997). Male circumcision and susceptibility to HIV infection in Tanzania. AIDS, 11, 73-80.

Weiss, H. A., Quigley, M. A., \& Hayes, R. J. (2000). Male circumcision and risk of HIV infection in sub-Saharan Africa: A systematic review and meta-analysis. AIDS, 14, 2261-2370.

World Health Organization. (2005). UNAIDS statement on South African trial findings regarding male circumcision and HIV Statement developed by the World Health Organization (WHO), the United Nations Population Fund (UNFPA), the United Nations Children's Fund (UNICEF) and the UNAIDS Secretariat, 26 July 2005. http://www. who.int/mediacentre/news/releases/2005/pr32/en/, Accessed on 15 March 2006. 